\title{
MAMMOGRAPHIC ABNORMALITIES AFTER CONSERVATIVE BREAST SURGERY WITH IMMEDIATE LIPOFILLING: CASE CONTROL STUDY
}

Andréa Pires Souto Damin¹, Heloise Neves, Jorge Villanova Biazús, Márcia Portela Melo², Ângela Erguy Zucatto ${ }^{2}$

${ }^{1}$ Graduate Program In Obstetrics And Gynecology, Universidade Federal do Rio Grande do Sul - Porto Alegre (RS), Brasil. ${ }^{2}$ Mastology Department, Hospital de Clínicas de Porto Alegre - Porto Alegre (RS), Brazil.

Introduction: Conservative breast surgery with immediate autologous fat grafting reconstruction (lipofilling) is emerging as a new technique for breast reconstruction. Concerns have been raised about mammographic abnormalities caused by immediate lipofilling. Lipofilling can lead to mammographic abnormalities, with diffuse microcalcifications as the most frequent, followed by oil cysts. The incidence of radiological abnormalities resulting from fat grafting varies significantly in the literature, ranging from 0 to $86 \%$. However, until now, no study has addressed the mammographic abnormalities found after conservative breast surgery with immediate lipofilling. Methods: This case-control study involved patients submitted to conservative breast surgery with or without immediate lipofilling in our facility between 2010 and 2013. Pathological and clinical characteristics of both groups were compared. The patients included underwent breast imaging tests with digital mammography every six months for two years. In each period, image changes were compared between the two groups. Results: Patients submitted to conservative breast surgery with immediate lipofilling were compared to those without immediate lipofilling. The mean volume grafted was $125 \pm 39 \mathrm{cc}$. Patients who underwent immediate lipofilling were younger than control patients ( 52.5 vs. 58.9 years, $\mathrm{p}=0.001$ ). Other pathological and clinical characteristics were not statistically different between the two groups. Mammographic findings revealed no significant differences in the frequency of nodules ( 0 vs. $8, \mathrm{p}=0.2$ ) and calcifications ( 30 vs. $92, \mathrm{p}=0.2$ ) between patients with and without lipofilling. However, patients submitted to immediate lipofilling presented a higher frequency of oil cysts $(6 \mathrm{vs.} 5, \mathrm{p}=0.01)$ and fat necrosis ( 3 vs. 1, p=0.03) after 24 months of radiotherapy. No patient was submitted to biopsy during the follow-up. Conclusions: This was the first study to show that lipofilling concomitantly with conservative breast surgery does not seem to induce significant radiological changes. These findings indicate that, from a radiological point of view, immediate lipofilling is safe and can be a new tool in breast oncoplasty. 